Accepted refereed manuscript of:

Reis DB, Rodriguez C, Acosta NG, Almansa E, Tocher DR, Andrade JP \& Sykes AV (2016) In vivo metabolism of unsaturated fatty acids in Sepia officinalis hatchlings, Aquaculture, 450, pp. 67-73.

DOI: 10.1016/j.aquaculture.2015.07.012

(C) 2015, Elsevier. Licensed under the Creative Commons AttributionNonCommercial-NoDerivatives 4.0 International http://creativecommons.org/licenses/by-nc-nd/4.0/ 


\section{In vivo metabolism of unsaturated fatty acids in Sepia officinalis}

\section{2 hatchlings}

3

4

5

6

8

9

D.B. Reis ${ }^{\mathrm{a}, \mathrm{b}, \mathrm{c}^{*}}$, C. Rodríguez ${ }^{\mathrm{b}}$, N.G. Acosta ${ }^{\mathrm{b}}$, E. Almansa $^{\mathrm{c}}$, D.R. Tocher ${ }^{\mathrm{d}}$, J.P. Andrade ${ }^{\mathrm{a}}$, and A.V. Sykes ${ }^{\mathrm{a}}$

${ }^{a}$ CCMAR - Centro de Ciências do Mar, Universidade do Algarve, Campus de Gambelas, 8005-139, Faro, Portugal.

bDepartamento de Biología Animal, Edafología y Geología, Universidad de La Laguna, Avenida Astrofísico Francisco Sánchez, 38206 La Laguna, Santa Cruz de Tenerife, Canary Islands, Spain.

${ }^{\mathrm{c}}$ Instituto Español de Oceanografía. Centro Oceanográfico de Canarias, Vía Espaldón $\mathrm{n}^{\circ} 1$, Dársena pesquera, Parcela ${ }^{\circ} 8, \mathrm{CP}: 38180$, Santa Cruz de Tenerife, Canary Islands, Spain.

${ }^{\mathrm{d}}$ Institute of Aquaculture, School of Natural Sciences, University of Stirling, Stirling FK9 4LA Scotland UK.

*Corresponding author at: CCMAR, Centro de Ciências do Mar do Algarve, Universidade do Algarve, Campus de Gambelas, 8005-139, Faro, Portugal. Tel: +351289800900 ext. 7430 Fax: +351289800069

Email address: dfbreis@ualg.pt; diana_b_reis@hotmail.com (D.B. Reis) 
The transition of Sepia officinalis culture to industrial large scale has been hampered due to bottlenecks related to the limited knowledge on nutritional physiology of the species. Determination of the endogenous ability of $S$. officinalis hatchlings to metabolise unsaturated fatty acids (FA) may provide new insight on the capability of hatchlings to biosynthesise different FA, as well as lipid classes containing essential fatty acids (EFA). In the present study, cuttlefish hatchlings were incubated with [1${ }^{14}$ C]FA including C18 FA (18:1n-9, 18:2n-6, 18:3n-3) and long-chain polyunsaturated fatty acids (LC-PUFA) (20:4n-6 (ARA), 20:5n-3 (EPA) or 22:6n-3 (DHA)), which were added individually as potassium salts bound to bovine serum albumin. As a result, it was possible to investigate the in vivo FA metabolism of $S$. officinalis hatchlings by following the incorporation of specific $\left[1-{ }^{14} \mathrm{C}\right] \mathrm{FA}$, which points to the suitability of this methodology to study lipid metabolism of newly hatched cephalopods. The majority of radioactivity incorporated was recovered esterified into polar lipids (PL). A pattern was detected, where $\left[1-{ }^{14} \mathrm{C}\right] \mathrm{DHA},\left[1-{ }^{14} \mathrm{C}\right] \mathrm{C} 18 \mathrm{FA}$ and their metabolic products were preferentially esterified into phosphatidylcholine, whereas $\left[1-{ }^{14} \mathrm{C}\right] \mathrm{ARA}$ and $\left[1-{ }^{14} \mathrm{C}\right] \mathrm{EPA}$ were mainly esterified into phosphatidylethanolamine. $\left[1-{ }^{14} \mathrm{C}\right] \mathrm{C} 18$ FA were the most transformed FA with several metabolites produced by elongation and possible desaturation being obtained. As a contrary the radioactivity incorporated into hatchling total lipid (TL) from supplemented $\left[1-{ }^{14} \mathrm{C}\right] \mathrm{LC}-\mathrm{PUFA}$ only one elongation product was recovered from the three substrates, except for $\left[1-{ }^{14} \mathrm{C}\right] \mathrm{ARA}$, where an unidentified product was also detected. The present in vivo results indicated that $S$. officinalis hatchlings may have capability for the first steps in the biosynthesis of ARA and EPA from 18:2n-6 and 18:3n-3, respectively, including the existence of a desaturase potentially involved. Nonetheless, considering the low desaturation rates detected, this process may not be sufficient to cover EFA demands during development of this species. Therefore, dietary ARA and EPA, as well as DHA, should be supplied during the hatchling stage of Sepia. While designing an inert diet, which ensures normal growth and development of this species during the hatchling stage, the C18 FA and LCPUFA levels and ratios should be considered, since the esterification pattern detected in the present study suggested competition between these FA for esterification into specific lipid classes. Moreover, considering the observed esterification pattern of LCPUFA into different lipid classes, it is likely that the DHA/EPA/ARA ratio, rather than 
DHA/EPA or EPA/ARA ratios, would be of great importance for $S$. officinalis hatchling development.

Keywords: DHA, EPA, ARA; Lipid metabolism; Radiolabelled substrates; Sepia officinalis hatchlings; Unsaturated fatty acids.

\section{Introduction}

The European cuttlefish (Sepia officinalis) has been recognized as a species with great potential for aquaculture, due to the short life cycle and high market prices which translate into short investment payback time (Sykes et al., 2014). Nonetheless, its culture could go beyond their use as human food. By-products of the food industry could be utilized in aquafeeds and pharmacological industries (Koueta et al., 2014), in addition to the use of these animals as a model for several research fields and for public exhibition in aquariums (see Sykes et al., 2014).

The rearing of $S$. officinalis has been successfully performed in several countries such as the U.S.A., Italy, France and Portugal. At the National Resource Centre for Cephalopods (U.S.A.) and the Centre of Marine Sciences (Portugal), several consecutive generations have been successfully cultured (Sykes et al., 2014). However, transition of technology to industry has been hampered due to three major bottlenecks: the dependence on live prey during the first part of the life cycle (the hatchling stage); the lack of an adequate artificial diet for all life stages; and the fact that full control of reproduction under rearing conditions has not yet been achieved (Sykes et al., 2006a; Villanueva et al., 2014).

During the hatchling stage, S. officinalis were reported to require live food (Sykes et al., 2006a), such as mysids and grass shrimp (Domingues et al., 2004; Sykes et al., 2006b). However, the use of these particular species makes cuttlefish culture not economically feasible (Domingues et al., 2010). Recently, Sykes et al. (2013) was able to feed $S$. officinalis with frozen grass shrimp from the first day after hatching, showing the potential of using an inert diet from birth. However, lower growth $(\sim 1 / 3$ of that achieved with live food) and higher mortality rates ( $20 \%$ more with frozen grass shrimp) were obtained. The differences in growth and survival were suggested to be associated with the maturation of the digestive system, possible changes in nutritional composition of prey or even the amount of prey captured (Sykes et al., 2013). Nonetheless, in order to design an artificial diet that would effectively replace live prey, it is important to 
understand the nutritional requirements and metabolic pathways of the species at this life stage.

Due to the low lipid content of cephalopods (Lee, 1994) and reports of their low capacity to metabolize lipids (O’Dor et al., 1984), lipid nutrition of S. officinalis has been neglected and only in recent years have some studies been devoted to this theme. Domingues et al. (2004) showed that either a quantitative or qualitative imbalance of prey lipid content could result in lower growth and survival rates in different life stages. However, as they grow in their natural environment, cuttlefish tend to change their diet with increased consumption of fish and decreased consumption of crustaceans (Castro and Guerra, 1990), possibly reflecting a change in metabolic requirements related to sexual maturity. The lipid composition of $S$. officinalis muscle normally presents high levels of phospholipids, cholesterol and polyunsaturated fatty acids (PUFA). For that reason, several authors suggested those nutrients as important for S. officinalis development and the necessity to provide a diet rich in those nutrients (Domingues et al., 2004, 2003; Koueta et al., 2002; Navarro and Villanueva, 2000).

Since formulated diets have been largely not accepted (Sykes et al., 2006a) or, if ingested, have resulted in poor growth and survival rates (Castro 1991, Castro and Lee 1994, Castro et al. 1993; Lee et al., 1991), the design of a diet to satisfy lipid requirements of this species cannot be performed only through doses-response studies. In this sense, studies of egg embryonic metabolism (Bouchaud and Galois, 1990; Sykes et al., 2009), lipid composition during hatchling starvation (Castro et al., 1992; Sykes et al., unpublished data), and feeding using prey with well characterized composition have been performed (Almansa et al., 2006; Domingues et al., 2003). Additionally, Reis et al. (2014a) recently reported the in vivo lipid metabolism in $O$. vulgaris hatchlings by incubating paralarvae with several ${ }^{14} \mathrm{C}$-labelled FA, where LC-PUFA were determined as essential for the normal development of the species. Moreover, considering the specific esterification patterns of the incubated FA, the same authors also suggested the necessity of adapting the dietary input of LC-PUFA in $O$. vulgaris diets. In this sense, a similar approach to that recently employed to determine the lipid requirements of $O$. vulgaris, could contribute to the understanding of lipid metabolism in cuttlefish. In the present study, the endogenous ability of $S$. officinalis hatchlings to biosynthesise FA and lipid classes containing those FA was determined by investigating the in vivo capability of the species to incorporate, esterify into different lipid classes, and modify unsaturated FA. 


\section{Materials \& Methods}

\subsection{Experimental animals}

All cuttlefish hatched on the same day from a single brood obtained from F4 cultured females reproducing at the Ramalhete Aquaculture Station (Ria Formosa, South of Portugal $-37^{\circ} 00^{\prime} 22.39^{\prime \prime} \mathrm{N} ; 7^{\circ} 58^{\prime} 02.69^{\prime \prime} \mathrm{W}$ ) and fed exclusively with frozen grass shrimp (Palaemonetes varians). Eggs $24 \mathrm{~h}$ after being laid were sent to IEO facilities in Tenerife and kept in $100 \mathrm{~L}$ circular fiberglass tanks in a flow-through seawater system. The embryonic development of eggs, as well as broodstock rearing, were assured using the technology described by Sykes et al. (2014).

Eggs were kept under a 100 lux incident light intensity and were subjected to a $13 \mathrm{~h}$ daylight cycle. Water temperature and dissolved oxygen, measured with a Tinytag Plus 2 (TGP-4020; Gemini Data Loggers Ltd., Chichester, West Sussex, UK), and salinity, measured with a Refractometer S/Mill-E (ATAGO, Tokyo, Japan), were determined in the morning on a daily basis. Water temperature was $21 \pm 0.69^{\circ} \mathrm{C}$, salinity was $36.8 \pm$ $0.14 \%$ and dissolved oxygen saturation level was $98.61 \pm 1.41 \%$.

\subsection{In vivo incubation with labelled $\left[1-{ }^{14} \mathrm{C}\right]$ fatty acids}

Twenty-eight newly hatched cuttlefish were incubated in 6-well flat-bottom tissue culture plates (Sarstedt AG \& CO., Nümbrecht, Germany), at a density of 1 hatchling per well, in $10 \mathrm{~mL}$ of seawater (36\%). Incubations were performed in quadruplicate at $21{ }^{\circ} \mathrm{C}$ for $6 \mathrm{~h}$ with gentle stirring and with $0.2 \mu \mathrm{Ci}(0.3 \mu \mathrm{M})$ of one $\left[1-{ }^{14} \mathrm{C}\right] \mathrm{FA}$ including 18:1n-9, 18:2n-6, 18:3n-3, 20:4n-6 (ARA), 20:5n-3 (EPA) or 22:6n-3 (DHA). [1$\left.{ }^{14} \mathrm{C}\right] \mathrm{FA}$ were individually added to separate wells as potassium salts bound to bovine serum albumin (BSA) as described by Ghioni et al. (1997). A control treatment of hatchlings was also assessed without the addition of $\left[1-{ }^{14} \mathrm{C}\right] \mathrm{FA}$. A $100 \%$ survival rate was obtained in all incubations.

156 After the incubation period, hatchlings were sacrificed in iced seawater and thoroughly 157 washed with filtered seawater to remove excess $\left[1-{ }^{14} \mathrm{C}\right] \mathrm{FA}$. Samples were stored at -80 ${ }^{\circ} \mathrm{C}$ until analysis. Total lipids (TL) were extracted with chloroform/methanol (2:1 by volume) according to the Folch method, as described by Christie (2003), and lipid content determined gravimetrically. The TL extracts were stored until analysis at $-20{ }^{\circ} \mathrm{C}$ in chloroform/methanol (2:1, by volume) with $0.01 \%$ butylated hydroxytoluene (BHT) 
as antioxidant, at a concentration of $10 \mathrm{mg} \cdot \mathrm{mL}^{-1}$ and under an inert atmosphere of nitrogen.

\subsection{Lipid classes and fatty acids composition of control samples}

Aliquots of $0.2 \mu \mathrm{l}$ of TL extract of hatchling control groups were used to determine lipid class $(\mathrm{LC})$ profiles $(\mathrm{n}=4)$. Classes were separated by single-dimensional doubledevelopment high-performance thin-layer chromatography (HPTLC) as previously described by Tocher and Harvie (1988) and quantified by charring, followed by calibrated densitometry using dual-wavelength flying spot scanner CS-9001PC (Shimadzu Co., Kyoto, Japan). Identification of individual LC was performed by running known standards (cod roe lipid extract and a mixture of single standards from BIOSIGMA S.r.1., Venice, Italy) on the same plates.

Fatty acid methyl esters (FAME) obtained by acid-catalysed transmethylation of $1 \mathrm{mg}$ of TL extracts, were purified by thin-layer chromatography (TLC) (Christie, 2003), and quantified using a TRACE-GC Ultra gas chromatograph (Thermo Fisher Scientific Inc., Waltham, Massachusetts, U.S.A.) equipped with a fused silica capillary column Supelcowax TM 10 (Sigma-Aldrich Co., St. Louis, Missouri, USA), on-column injector and flame ionization detector. Individual FAME were confirmed by GC-MS chromatography (DSQ II, Thermo Fisher Scientific Inc., Waltham, Massachusetts, U.S.A.).

\subsection{Incorporation of radioactivity into total lipids}

In order to determine the radioactivity incorporated into hatchling TL, an aliquot of 0.1 mg of TL extract was transferred to scintillation vials and radioactivity quantified in an LKB Wallac 1214 Rackbeta liquid scintillation $\beta$-counter (PerkinElmer Inc., Waltham, Massachusetts, U.S.A.). Results in dpm were converted into pmoles per mg of protein

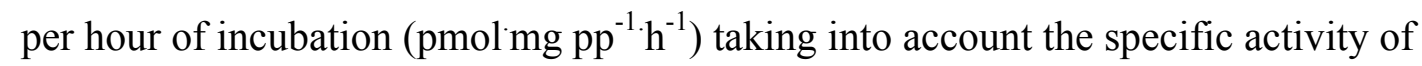
each substrate and hatchling lipid and protein contents. The protein content of hatchlings $(n=4)$ was determined according to Lowry et al. (1951).

\subsection{Esterification of radiolabelled FA into lipid classes}

In order to define the esterification pattern of each $\left[1-{ }^{14} \mathrm{C}\right] \mathrm{FA}$ into different $\mathrm{LC}, 0.1 \mathrm{mg}$ of TL extract was applied to HPTLC plates. LC were separated by single-dimensional double-development HPTLC as described previously (Tocher and Harvie, 1988). The 
esterification pattern of each $\left[1-{ }^{14} \mathrm{C}\right] \mathrm{FA}$ into a given $\mathrm{LC}$ was determined by image analysis following the method described by Reis et al. (2014a).

\subsection{Transformation of radiolabelled FA}

To determine the metabolism of radiolabelled substrates by desaturation/elongation, a sample of $1.3 \mathrm{mg}$ of TL was subjected to acid-catalysed transmethylation to prepare FAME as detailed above. FAME separation was achieved using TLC plates impregnated with a solution of $2 \mathrm{~g}$ silver nitrate in $20 \mathrm{~mL}$ acetonitrile followed by activation at $110^{\circ} \mathrm{C}$ for $30 \mathrm{~min}$. The plates were fully developed in toluene/acetonitrile (95:5, v/v), which resolved the FAME into discrete bands based on both degree of unsaturation and chain length (Wilson and Sargent, 1992). FAME identification and quantification was performed by image analysis following the method described by Reis et al. (2014a).

\subsection{Reagents}

$\left[1-{ }^{14} \mathrm{C}\right] \mathrm{C} 18 \mathrm{FA}$ were purchased as free FA form, dissolved in ethanol from PerkinElmer, Inc. (Waltham, Massachusetts, U.S.A.). $\left[1-{ }^{14} \mathrm{C}\right] \mathrm{LC}-\mathrm{PUFA}$ were also purchased as free FA from American Radiolabeled Chemicals, Inc. (St. Louis, Missouri, U.S.A.). BSA was purchased from Sigma-Aldrich Co. (St. Louis, Missouri, U.S.A.). TLC plates $(20 \times$ $20 \mathrm{~cm} \times 0.25 \mathrm{~mm}$ ) were purchased form Macherey-Nagel GmbH \& Co. KG (Düren, Germany). HPTLC plates, $(10 \times 10 \mathrm{~cm} \times 0.15 \mathrm{~mm})$ pre-coated with silica gel 60 (without fluorescent indicator), were purchased from Merck KGaA (Düsseldorf, Germany). OptiPhase "HiSafe" 2 scintillant liquid was purchased from PerkinElmer, Inc. (Waltham, Massachusetts, U.S.A.). Organic solvents used were of reagent grade and were purchased from Merck KGaA (Düsseldorf, Germany), Sigma-Aldrich Co. (St. Louis, Missouri, U.S.A.) and Panreac Química S.L.U. (Barcelona, Spain).

\subsection{Statistical analysis}

Results are presented as means $\pm \mathrm{SD}(\mathrm{n}=4)$. For all statistical tests, $p$ values $<0.05$ were considered statistically different. Data were checked for normal distribution with the one-sample Shapiro-Wilk test (Zar, 1999), as well as for homogeneity of the variances with the Levene test (Zar, 1999). Arcsine square root transformation was applied to all data expressed as percentage (Fowler et al., 1998). Incorporation and 
esterification comparisons between the six FA means and within $\left[1-{ }^{14} \mathrm{C}\right] \mathrm{C} 18$ FA $(18: 1 \mathrm{n}-$ 9, 18:2n-6, 18:3n-3) and $\left[1-{ }^{14} \mathrm{C}\right]$ LC-PUFA (ARA, EPA, DHA), as well as data of unmodified FA recovery between the six FA means were performed by one-way analysis of variance (ANOVA) followed by a Tukey's post hoc test (Zar, 1999). When normal distribution was not achieved, data were subjected to a Kruskal-Wallis nonparametric test, and when the homogeneity of variances were not achieved, data were subjected to the Welch robust ANOVA, followed by a Games-Howell non-parametric multiple comparison test (Zar, 1999). The statistical analysis was performed using the IBM SPSS statistics 22.0 (IBM Co., Armonk, New York, U.S.A.).

\section{Results}

\subsection{Lipid composition of hatchlings}

The TL content and LC composition of $S$. officinalis hatchlings are presented in Table 1. The TL profile of S. officinalis hatchlings consisted mainly of polar lipids (PL; 58.1 \pm $1.0 \%$ ) and cholesterol (32.2 $\pm 2.1 \%)$. Within PL, phosphatidylcholine (PC; $24.0 \pm 1.9$ $\%)$ and phosphatidylethanolamine (PE; $18.9 \pm 1.0 \%)$ were the main classes. On the other hand, triacylglycerols (TAG; $6.3 \pm 1.1 \%$ ) were the second major class within neutral lipids (NL; $41.9 \pm 1.0 \%)$.

Table 2 shows the FA profile of $S$. officinalis hatchlings. Hatchlings were particularly rich in DHA $(21.5 \pm 1.7 \%$ of total FA), 16:0 (20.4 $\pm 1.6 \%)$ and EPA $(16.4 \pm 1.0 \%)$. ARA, 18:1n-9 and 18:2n- 6 only accounted for $1.3 \pm 0.4 \%, 1.5 \pm 0.3 \%$ and $0.6 \pm 0.1 \%$ of total FA, respectively. The polyunsaturated FA content of hatchlings was $43.1 \pm 2.6$ $\%$ of total FA, while LC-PUFA accounted for $41.8 \pm 2.7 \%$ of total FA. The DHA/EPA ratio of hatchlings was $1.3 \pm 0.0$, while the EPA/ARA ratio was $13.4 \pm 3.9$.

\subsection{Incorporation of radiolabelled FA into TL and its distribution among $L C$}

The incorporation of radiolabelled FA into $S$. officinalis hatchling TL and its distribution among LC is presented in Table 3. Within the C18 FA, $\left[1-{ }^{14} \mathrm{C}\right] 18$ : $1 \mathrm{n}-9$ was the least incorporated FA $(p<0.05)$. $\left[1-{ }^{14} \mathrm{C}\right] 18: 3 \mathrm{n}-3$ and $\left[1-{ }^{14} \mathrm{C}\right] \mathrm{ARA}$ were the most incorporated FA, although not statistically different from $\left[1-{ }^{14} \mathrm{C}\right] 18: 2 \mathrm{n}-6$ or $\left[1-{ }^{14} \mathrm{C}\right] \mathrm{EPA}$. All $\left[1-{ }^{14} \mathrm{C}\right] \mathrm{FA}$ substrates incorporated were highly esterified into LC, with less than 2.5 $\%$ of radioactivity being recovered as free fatty acids (FFA). The majority of radioactivity incorporated was recovered esterified into PL, with over $90 \%$ of the LCPUFA substrates being esterified into this lipid fraction. The proportion of radioactivity 
recovered in TAG was higher for the $\left[1-{ }^{14} \mathrm{C}\right] \mathrm{C} 18$ FA than for LC-PUFA $(p<0.05)$. Nonetheless, $\left[1-{ }^{14} \mathrm{C}\right] \mathrm{C} 18$ FA were preferentially esterified into $\mathrm{PC}$ and phosphatidylserine (PS). The esterification of $\left[1-{ }^{14} \mathrm{C}\right] \mathrm{ARA}$ and $\left[1-{ }^{14} \mathrm{C}\right] \mathrm{EPA}$ into the different LC was similar, showing a preferential esterification into $\operatorname{PE~}(p<0.05)$, followed by $\mathrm{PC}>\mathrm{PS}>$ phosphatidylinositol $(\mathrm{PI})$. On the other hand, $\left[1-{ }^{14} \mathrm{C}\right] \mathrm{DHA}$ was mainly esterified into PC $(70.6 \pm 4.8 \%$ of radioactivity incorporated; $p<0.05)$. The esterification pattern of all the $\left[1-{ }^{14} \mathrm{C}\right] \mathrm{C} 18 \mathrm{FA}$ substrates was similar, with preferential esterification into $\mathrm{PC}>\mathrm{PS}>\mathrm{PE}>\mathrm{TAG}$.

\subsection{Transformation of radiolabelled fatty acids}

The transformation of incorporated $\left[1-{ }^{14} \mathrm{C}\right] \mathrm{FA}$ substrates into other FA metabolites is presented in Table 4. $\left[1-{ }^{14} \mathrm{C}\right] \mathrm{C} 18$ FA were the most transformed FA $(p<0.05)$ with several metabolic products being detected. For instance, $\left[1-{ }^{14} \mathrm{C}\right] 18: 1 \mathrm{n}-9$ was elongated to $20: 1 n-9(42.0 \pm 5.6 \%)$ and $22: 1 n-9(12.7 \pm 1.9 \%)$. Although the band corresponding to $22: 1 \mathrm{n}-9$ appeared to be split, unequivocal confirmation of the presence of two single bands was not possible. Similar to $\left[1-{ }^{14} \mathrm{C}\right] 18: 1 \mathrm{n}-9,\left[1-{ }^{14} \mathrm{C}\right] 18: 2 \mathrm{n}-6$ and $\left[1-{ }^{14} \mathrm{C}\right] 18: 3 \mathrm{n}-3$ presented two elongation products. In addition, potential desaturation products from the three $\left[1-{ }^{14} \mathrm{C}\right] \mathrm{C} 18 \mathrm{FA}$ substrates were also detected. A second potential desaturation product from $\left[1-{ }^{14} \mathrm{C}\right] 18: 2 \mathrm{n}-6$ was also observed, although it was not possible to confirm its identity. Of the radioactivity incorporated into hatchlings TL from supplemented [1$\left.{ }^{14} \mathrm{C}\right] \mathrm{LC}-\mathrm{PUF} A$ only one elongation product was recovered, except for $\left[1-{ }^{14} \mathrm{C}\right] \mathrm{ARA}$ where an unidentified product, obtained by a potential desaturation, was also detected (Table 4).

\section{Discussion}

In the present study, similar to that previously reported for $O$. vulgaris (Reis et al., 2014a), it was possible to investigate the in vivo FA metabolism of S. officinalis hatchlings by following the incorporation of specific $\left[1-{ }^{14} \mathrm{C}\right] \mathrm{FA}$. The obvious incorporation of all the substrates, points to the suitability of this methodology to study lipid metabolism of newly hatched cephalopods even though it is not clear yet if the FA are being incorporated through drinking activity and/or the integument. As in other marine invertebrates, the epithelial tissues of cephalopods possess microvilli-like absorptive epithelia with the capacity to uptake organic compounds directly from the environment (de Eguileor et al., 2000). 
As previously reported by Navarro and Villanueva (2000), cuttlefish hatchlings presented high contents of PC, PE and cholesterol, with 16:0, 18:0, EPA and DHA being the most abundant FA. High contents of these LC and FA were also detected in cuttlefish eggs (Sykes et al., 2009), and in juvenile and adult mantle (Almansa et al., 2006; Ferreira et al., 2010; Sinanoglou and Miniadis-Meimaroglou, 2000, 1998). The consistent high levels of these LC and FA in lipid profiles of S. officinalis may reflect their importance during lifespan of this species. Based on that, Navarro and Villanueva (2000) suggested that $S$. officinalis requires a diet rich in PUFA, PL and cholesterol, with moderate content of NL for normal development.

In cuttlefish hatchings, different from that previously reported for $O$. vulgaris hatchlings where a specifically higher incorporation of ARA into TL was observed (Reis et al., 2014a), $\left[1-{ }^{14} \mathrm{C}\right] \mathrm{ARA}$ was not preferentially incorporated compared to EPA or C18PUFA. The ARA levels found in S. officinalis tissues (Almansa et al., 2006; Navarro and Villanueva, 2000; Sykes et al., 2009) are normally lower than those detected in O. vulgaris (Monroig et al., 2012a; Navarro and Villanueva, 2000; Reis et al., 2014a, 2014b; Viciano et al., 2011), which could point to different requirements for this FA between these species. In contrast, the EPA content found in the present study was generally similar to that reported for O. vulgaris hatchlings (see Navarro and Villanueva, 2000; Reis et al., 2014a, 2014b).

Interestingly, and similar to that described by Reis et al. (2014a) for O. vulgaris hatchlings, cuttlefish hatchlings presented a similar pattern of esterification of labelled ARA and EPA into different LC, with the majority of the incorporated LC-PUFA being recovered in PE. However, contrary to the results of the earlier study on octopus, both EPA and ARA also showed high esterification into PC and PS in cuttlefish. Sinanoglou and Miniadis-Meimaroglou (2000) determined the FA content of PE and PC of $S$. officinalis mantle, and observed a high content of EPA and ARA in PE. On the other hand, a high level of DHA was observed in PC by the same authors. Similarly, in the present study, DHA was mainly esterified into PC (70.6 $\pm 4.8 \%$ of incorporated [1$\left.\left.{ }^{14} \mathrm{C}\right] \mathrm{DHA}\right)$. When comparing the esterification pattern of $\left[1-{ }^{14} \mathrm{C}\right] \mathrm{LC}-\mathrm{PUFA}$ in $S$. officinalis hatchlings with that of $O$. vulgaris (Reis et al., 2014a), there appears to be lower specificity in the esterification of FA into specific LC in cuttlefish. Therefore, the DHA/EPA/ARA ratio would be of greater importance in $S$. officinalis development, rather than the EPA/ARA ratio, which appears to have greater importance for $O$. vulgaris paralarvae. 
Sinanoglou and Miniadis-Meimaroglou (1998) detected a high content of monounsaturated FA in the NL fraction of S. officinalis mantle. In the present study, the proportion of radioactivity esterified into NL was slightly higher for $\left[1-{ }^{14} \mathrm{C}\right] 18: 1 \mathrm{n}-9$, $\left(19.4 \pm 3.4 \%\right.$ of incorporated $\left.\left[1-{ }^{14} \mathrm{C}\right] 18: 1 \mathrm{n}-9\right)$. Nonetheless, when compared to [1$\left.{ }^{14} \mathrm{C}\right]$ LC-PUFA, high proportions of $\left[1-{ }^{14} \mathrm{C}\right] 18: 2 \mathrm{n}-6$ and $\left[1-{ }^{14} \mathrm{C}\right] 18: 3 \mathrm{n}-3$ were also recovered in NL. This was similar to the data described for $O$. vulgaris hatchling metabolism (Reis et al., 2014a), where $\left[1-{ }^{14} \mathrm{C}\right] \mathrm{C} 18$ FA were highly esterified into NL, as well as into PC. Moreover, in the present study, there was a significant proportion of [1$\left.{ }^{14} \mathrm{C}\right] \mathrm{C} 18$ FA esterified into PS, when compared to $\left[1-{ }^{14} \mathrm{C}\right]$ LC-PUFA. Again, similar to esterification pattern of $\left[1-{ }^{14} \mathrm{C}\right] \mathrm{LC}-\mathrm{PUFA}$, when comparing $S$. officinalis hatchlings with O. vulgaris (Reis et al., 2014a), lower specificity in the esterification of $\left[1-{ }^{14} \mathrm{C}\right] \mathrm{C} 18 \mathrm{FA}$ substrates into particular LC was observed in cuttlefish.

It is interesting to note that all substrates were mainly esterified into the major LC of $S$. officinalis hatchlings (PC and PE, followed by PS and TAG). However, a pattern was observed, where $\left[1-{ }^{14} \mathrm{C}\right] \mathrm{DHA}$, and $\left[1-{ }^{14} \mathrm{C}\right] \mathrm{C} 18 \mathrm{FA}$ were preferentially esterified into $\mathrm{PC}$, whereas $\left[1-{ }^{14} \mathrm{C}\right] \mathrm{ARA}$ and $\left[1-{ }^{14} \mathrm{C}\right] \mathrm{EPA}$ were mainly esterified into PE. In this respect, and considering the competition between these FA for several enzyme activities (Sargent et al., 1999), it seems important to ensure an adequate dietary input of those FA. For that, while designing a suitable diet for S. officinalis hatchlings ARA/EPA/DHA, as well as C18 FA/LC-PUFA ratios must be considered. Moreover, future studies are necessary in a way to elucidate the capacity of this animals to translocate dietary EFA within phospholipids and between TAG and phospholipids. When analysing the variation of FA composition during embryonic development of $S$. officinalis, Sykes et al. (2009) observed an increase in the n-9 FA fraction, particularly 20:1n-9, suggesting possible de novo synthesis of these FA. Moreover, the amount of 20:2n-6 and 20:3n-3 also increased during embryogenesis in wild eggs (Sykes et al., 2009). The results of the present study confirmed a high capability of $S$. officinalis hatchlings to elongate $\left[1-{ }^{14} \mathrm{C}\right] \mathrm{C} 18 \mathrm{FA}$ to $\mathrm{C} 20 \mathrm{FA}$. In addition, a second elongation step was also noted, as a C22 FA band was obtained from all the $\left[1-{ }^{14} \mathrm{C}\right] \mathrm{C} 18 \mathrm{FA}$ substrates. However, only 22:1n-9 was previously reported by Sykes et al. (2009) in cuttlefish eggs and by Dumont et al. (1992) in the central nervous system of this species, albeit in insignificant amounts.

The capacity of cuttlefish hatchlings to elongate FA was further confirmed with the [1${ }^{14}$ C]LC-PUFA substrates. Monroig et al. (2012b) have reported the functional 
characterization of an elongase of very long-chain FA (Elovl) gene in O. vulgaris, which showed the capacity of this species to convert C18 and C20 PUFA substrates to their corresponding 2C elongated products, although no activity towards C22 PUFA was detected with this Elovl. More recently, Monroig et al. (2013) reported the functional characterization of a second Elovl, which suggested octopus had the capability to elongate C22 PUFA. The present results, and those obtained in octopus (Reis et al., 2014a), showed elongation activity towards DHA. Interestingly, this activity was not detected in any C22 FA products obtained from $\mathrm{C} 18$ or C20 FA substrates. Considering, the low amount of radioactivity recovered as $\mathrm{C} 22 \mathrm{FA}$, and the apparent low elongation activity on these FA, (less than $6 \%$ of $\left[1-{ }^{14} \mathrm{C}\right] \mathrm{DHA}$ incorporated was recovered as 24:6n-3), it is possible that the band obtained would be unnoticed on a TLC plate. Additionally, as mentioned above, in some replicates of [1$\left.{ }^{14} \mathrm{C}\right] 18: 1 \mathrm{n}-9$, the bands corresponding to C22 FA appeared to be split. However, an unequivocal and accurate confirmation of its existence and identity was not possible. In contrast to fish, where more efficient elongation of n-3 is reported (Agaba et al., 2005; Monroig et al., 2012c; Morais et al., 2011), in the present study higher activity towards n-6 FA substrates was detected. Thus, 18:2n-6 and 20:4n-6 were transformed at higher rates than the corresponding n-3 FA, namely 18:3n-3 and 20:5n-3, respectively. Preferential elongation activity towards n-6 FA rather than n-3 FA was also previously reported in O. vulgaris (Monroig et al., 2012b; Reis et al., 2014a). Nonetheless, considering the incorporated radioactivity into $S$. officinalis hatchlings TL, the absolute value of elongated FA was similar between n-3 and n-6 FA $(7.2 \pm 1.1$ and $7.6 \pm 0.3$ pmoles.mg $\mathrm{pp}^{-1} \cdot \mathrm{h}^{-1}$ for $18: 3 \mathrm{n}-3$ and $18: 2 \mathrm{n}-6$, respectively; and $0.3 \pm 0.0$ and $0.4 \pm 0.2$ pmoles.mg $\mathrm{pp}^{-1} \cdot \mathrm{h}^{-1}$ for EPA and ARA, respectively).

Almansa et al. (2006) suggested the existence of active n-6 LC-PUFA metabolism in juvenile and maturing cuttlefish as biosynthesis of 22:5n-6 from ARA was implied. In animals, 22:5n-6 has been considered as the final end product of the desaturation pathways of n-6 FAs (Tocher et al., 1998). The synthesis of this FA from ARA involves one of two possible metabolic pathways: the "Sprecher pathway", which comprises two sequential elongations of ARA to $24: 4 n-6$, followed by a $\Delta 6$ desaturation and one round of peroxisomal $\beta$-oxidation ( $\Delta 4$-independent pathway; Sprecher, 2000); or a direct route involving elongation of ARA to $22: 4 n-6$ followed by a $\Delta 4$ desaturation (Li et al., 2010). For many years the activity of $\Delta 4$ fatty acyl desaturase enzyme (Fad) was not demonstrated in vertebrates, and it was assumed that DHA was synthesised by the $\Delta 4$ - 
independent pathway (Tocher et al., 1998). However, recent studies have identified the activity of a $\Delta 4 \mathrm{Fad}$, not just in lower eukaryotes (Pereira et al., 2003), but also in some teleost fish species (Li et al., 2010; Morais et al., 2015, 2012; Fonseca-Madrigal et al., 2014). As previously mentioned, the present study confirmed the elongation of [1${ }^{14}$ C]ARA to $22: 4 n-6$. Moreover, another unidentified metabolic product from [1$\left.{ }^{14} \mathrm{C}\right] \mathrm{ARA}$ was observed, which could support the previous suggestion of Almansa et al. (2006). However, a similar band detected in O. vulgaris hatchling $\left[1-{ }^{14} \mathrm{C}\right] \mathrm{ARA}$ metabolism was determined as a metabolic product other than a FA (Reis et al., unpublished data). Furthermore, no similar band was obtained from $\left[1-{ }^{14} \mathrm{C}\right] \mathrm{EPA}$. Interestingly, a similar metabolic product to that detected from $\left[1-{ }^{14} \mathrm{C}\right] \mathrm{ARA}$, was also obtained from $\left[1-{ }^{14} \mathrm{C}\right] 18: 2 \mathrm{n}-6$. Since there was no evidence for ARA production from $\left[1-{ }^{14} \mathrm{C}\right] 18: 2 \mathrm{n}-6$, it would be unexpected that this band corresponds to $22: 5 \mathrm{n}-6$. Although it was not possible to identify the identity of this band, it is important to mention its appearance when n-6 FA were incubated, since it may possibly indicate a different requirement of cephalopods for n-6 FA.

The synthesis of EPA and ARA from 18:3n-3 and 18:2n-6, respectively, requires the activity of a $\Delta 6 \mathrm{Fad}$, followed by elongation, and then a second Fad introducing a double bond at the $\Delta 5$ position of the elongated FA (Cook, 1996). However, in some marine fish species, the $\Delta 6$ enzyme also presents a $\Delta 8$ activity and, so the first two steps could be reversed in order (Monroig et al., 2011). In the present study, according to the position of co-running standards, the potential resultant desaturation products from [1$\left.{ }^{14} \mathrm{C}\right] \mathrm{C} 18 \mathrm{FA}$ substrates might involve a $\Delta 6$ desaturation. Nonetheless, unequivocal identification of the desaturation products obtained from C18 FA (18:2n-9, 18:3n-6 and 18:4n-3) would be difficult, since the route involving an elongation followed by $\Delta 8$ desaturation (producing 20:2n-9, 20:3n-6 and 20:4n-3) cannot be eliminated. In this sense, the characterization of the enzymes involved in PUFA biosynthesis of cuttlefish would be an invaluable tool.

Functional characterization in recombinant yeast showed that $O$. vulgaris possessed a Fad that exhibited $\Delta 5$ desaturation activity towards PUFA substrates (Monroig et al., 2012a). Recently, similar results were also obtained regarding Fad genes of $S$. officinalis hatchlings (Monroig et al., 2013). However, no $\Delta 5$ Fad activity was detected in the present study. Despite this, the present data do not rule out a possible $\Delta 5 \mathrm{Fad}$ activity in ARA or EPA biosynthesis in vivo. The low desaturation rates observed in the present 
study and the low $\Delta 5$ Fad activity on PUFA substrates, reported by Monroig et al. (2012a), could explain the absence of radiolabelled ARA or EPA bands.

In summary, the methodology employed in the present study allows to investigate the in vivo FA metabolism of $S$. officinalis hatchlings by following the tissue incorporation of specific $\left[1-{ }^{14} \mathrm{C}\right] \mathrm{FA}$, and points out to the suitability of this methodology to study lipid metabolism of newly hatched cephalopods. The present results may indicate the possibility that $S$. officinalis hatchlings have in vivo capability for the first steps of ARA and EPA biosynthesis from 18:2n-6 and 18:3n-3, respectively, including a possible desaturase. Nonetheless, considering the low desaturation rates detected, this process would not be sufficient to satisfy the demands for normal development of this species. Therefore, both ARA and EPA, along with DHA, must be supplied in the diet at least during the hatchling stage. In addition, the esterification specificity for DHA into PC and of ARA and EPA into PE and PC, detected in the present study, could be hampered by competition between them and also between C18 FA and LC-PUFA. Therefore, in order to design an efficient inert diet that would ensure normal growth and development of this species during early life stages, DHA/EPA/ARA ratio, rather than EPA/ARA or EPA/DHA ratios and also $\mathrm{C} 18 \mathrm{FA}$ and LC-PUFA ratio, must be considered to reflect the EFA requirements for $S$. officinalis hatchlings development. Nonetheless, future studies are necessary in a way to elucidate the capacity of this animals to translocate dietary EFA within phospholipids and between TAG and phospholipids.

\section{Aknowledgements}

The use of animals in the experiments of this work were in accordance with the EU Directive 2010/63/EU for animal experimentation.

This work was partially supported by projects OCTOPHYS (AGL 2010-22120-CO3) funded by the Spanish Government, SEPIABREED ((PTDC/MAR/120876/2010) funded by Fundação para a Ciência e a Tecnologia (FCT) and SEPIATECH (31-03-05FEP-2) funded by the Portuguese Government Program PROMAR, which is co-funded by the EU Fisheries Fund. D.B. Reis (SFRH/BD/76863/2011) wish to thank FCT for her grant. A.V. Sykes is funded by FCT through Programa Investigador FCT 2014 (IF/00576/2014). Dr. Rodríguez is a member of the ITB of La Laguna University.

\section{References}


Agaba, M.K., Tocher, D.R., Dickson, C.A., Zheng, X., Dick, J.R., Teale, A.J., 2005. Cloning and functional characterisation of polyunsaturated fatty acid elongases from marine and freshwater teleost fish. Comp. Biochem. Physiol. B 142, 342352.

Almansa, E., Domingues, P., Sykes A., Tejera T., Lorenzo A., Andrade J.P., 2006. The effects of feeding with shrimp or fish fry on growth and mantle lipid composition of juvenile and adult cuttlefish (Sepia officinalis). Aquaculture 256, 403-413.

Bouchaud, O., Galois, R., 1990. Utilization of egg-yolk lipids during the embryonic development of Sepia officinalis L. in relation to temperature of the water. Comp. Biochem. Physiol. B 97, 611-615.

Castro, B.G., 1991. Can Sepia officinalis be reared on artificial food? Mar. Behav. Physiol. 19, 35-38.

Castro, B.G., Lee, P.G., 1994. The effects of semi-purified diets on growth and condition of Sepia officinalis L. (Mollusca: Cephalopoda). Comp. Biochem. Physiol. A 109, 1007-1016.

Castro, B.G., Guerra, A., 1990. The diet of Sepia officinalis (Linneaus, 1758) and Sepia elegans (Blainville, 1827) (Cephalopoda, Sepioidea) from the Ria of Vigo (NW Spain). Sci. Mar. 54, 375-388.

Castro, B.G., Garrido, J.L., Sotelo, C.G., 1992. Changes in composition of digestive gland and mantle muscle of the cuttlefish Sepia officinalis during starvation. Mar. Biol. 114, 11-20.

Castro, B.G., DiMarco, F.P., DeRusha, R.H., Lee, P.G., 1993. The effects of surimi and pelleted diets on the laboratory survival, growth and feeding rate of the cuttlefish Sepia officinalis L. J. Exp. Mar. Biol. Ecol. 170, 241-252.

Christie, W.W., 2003. Lipid Analysis, third ed. The Oily Press, Bridgewater, UK.

Cook, H.W., 1996. Fatty acids desaturation and chain elongation in eukaryontes, in: Vance, D.E., Vance, J. (Eds.), Biochemistry of Lipids, Lipoproteins and Membranes. Elsevier, Amsterdam, pp. 129-152.

de Eguileor, M., Leonardi, M.G., Grimaldi, A., Tettamanti, G., Fiandra, L., Giordana, B., Valvassori, R., Lanzavecchia, G., 2000. Integumental amino acid uptake in carnivorous predator mollusc (Sepia officinalis, Cephalopoda). Tissue Cell 32, 389-398. 
Domingues, P.M., Poirier, R., Dickel, L., Almansa, E., Sykes, A., Andrade, J., 2003b. Effects of culture density and live prey on growth and survival of juvenile cuttlefish, Sepia officinalis. Aquacult. Int. 11, 225-242.

Domingues, P., Sykes, A., Sommerfield, A., Almansa, E., Lorenzo, A., Andrade, P., 2004. Growth and survival of cuttlefish, Sepia officinalis (Linnaeus, 1758) of different ages fed crustaceans and fish. Effects of frozen and live prey. Aquaculture 229, 239-254.

Domingues, P., Garcia, S., Hachero-Cruzado, I., Lopez, N., Rosas, C., 2010. The use of alternative prey (crayfish, Procambarus clarki, and hake, Merlucius gayi) to culture Octopus vulgaris (Cuvier 1797). Aquacult. Int. 18, 487-499.

Dumont, E., Chichery M.P., Chichery R., Nouvelot A., 1992. Analysis of the fatty acids in the central nervous system of Sepia officinalis. Comp. Biochem. Physiol. B $101,341-344$.

Ferreira A., Marquez L., Almansa E., Andrade J.P., Lorenzo A., Domingues P.M., 2010. The use of alternative diets to culture juvenile cuttlefish, Sepia officinalis: effects on growth and lipid composition. Aquacult. Nutr. 16, 262-275.

Fonseca-Madrigal, J., Navarro, J.C., Hontoria, F., Tocher, D.R., Martínez-Palacios, C.A., Monroig, O., 2014. Diversification of substrate specificities in teleostei Fads2: characterization of $\Delta 4$ and $\Delta 6 \Delta 5$ desaturases of Chirostoma estor. J. Lipid Res. 55, 1408-1419.

Fowler, J., Cohen, L., Jarvis, P., 1998. Practical statistics for field biology, second ed. John Wiley \& Sons, New York.

Ghioni, C., Tocher, D.R., Sargent, J.R., 1997. The effect of culture on morphology, lipid and fatty acid composition, and polyunsaturated fatty acid metabolism of rainbow trout (Oncorhynchus mykiss) skin cells. Fish Physiol. Biochem. 16, 499513.

Koueta, N., Boucaud-Camou, E., Noel, B., 2002. Effects of enriched natural diet on survival and growth of juvenile cuttlefish Sepia officinalis L. Aquaculture 203, 293-310.

Koueta, N., Viala, H., Le Bihan, E., 2014. Applications, Uses and By-products from Cephalopods, in: Iglesias, J., Fuentes, L., Villanueva, R., (Eds.) Cephalopod Culture. Springer, Heidelberg, pp. 131- 147.

Lee, P., Forsythe, J., DiMarco, F., DeRusha, R. \& Hanlon, R., 1991. Initial palatability and growth trials on pelleted diets for cephalopods. Bull. Mar. Sci., 49, 362-372 
Lee, P.G., 1994. Nutrition of cephalopods: fuelling the system. Mar. Freshw. Behav. Physiol. 25, 35-51.

Li Y, Monroig O, Zhang L, Wang S, Zheng X, Dick J.R., You C., Tocher D.R., 2010. Vertebrate fatty acyl desaturase with $\Delta 4$ activity. Proc. Natl. Acad. Sci. USA. 107, $16840-16845$.

Lowry, O.H., Rosebrough, N.J., Farr, A.L., Randall, R.J., 1951. Protein measurement with folin phenol reagent. J. Biol. Chem. 193, 265-275.

Monroig, O., Li Y., Tocher D.R., 2011. Delta-8 desaturation activity varies among fatty acyl desaturase of teleost fish: High activity in delta-6 desaturase of marine species. Comp. Biochem. Physiol. B 159, 206-213.

Monroig, O., Navarro, J.C., Dick, R., Alemany, F., Tocher, D.R., 2012a. Identification of a $\Delta 5$-like Fatty Acyl Desaturase from the Cephalopod Octopus vulgaris (Cuvier 1797) Involved in the Biosynthesis of Essential Fatty Acids. Mar. Biotechnol. 14, 411-422.

Monroig, O., Guinot, D., Hontoria, F., Tocher, D.R., Navarro, J.C., 2012b. Biosynthesis of essential fatty acids in Octopus vulgaris (Cuvier, 1797): Molecular cloning, functional characterization and tissue distribution of a fatty acyl elongase. Aquaculture 360-361, 45-53.

Monroig, O., Wang, S., Zhang, L., You, C., Tocher, D.R., Li, Y., 2012c. Elongation of long-chain fatty acids in rabbitfish Siganus canaliculatus: Cloning, functional characterisation and tissue distribution of Elovl5- and Elovl4-like elongases. Aquaculture 350-353, 63-70.

Monroig, O., Tocher R.D., Navarro, J.C., 2013. Biosynthesis of Polyunsaturated Fatty Acids in Marine Invertebrates: Recent Advances in Molecular Mechanisms. Mar. Drugs 11, 3998-4018.

Morais, S., Castanheira, F., Martinez-Rubio, L., Conceição L.E.C., Tocher, D.R., 2012. Long chain polyunsaturated fatty acids synthesis in marine vertebrate: Ontogenetic and nutritional regulation of fatty acyl desaturase with $\Delta 4$ activity. Biochim. Biophys. Acta 1821, 660-671.

Morais, S., Mourente, G., Martínez, A., Gras, N., Tocher, D.R., 2015. Docosahexaenoic acid biosynthesis via fatty acyl elongase and $\Delta 4$-desaturase and its modulation by dietary lipid level and fatty acid composition in marine vertebrate. Biochim. Biophys. Acta 1851, 588-597. 
Navarro, J., Villanueva, R., 2000. Lipid and fatty acid composition of early stages of cephalopods: an approach to their lipid requirements. Aquaculture 183, 161-177.

O’Dor, R.K., Mangold, K., Boucher-Rodoni, R., Wells, M.J. \& Wells, J., 1984. Nutrient absorption, storage and remobilization in Octopus vulgaris. Mar. Behav. Physiol., $11,239-258$.

Pereira, S.L., Leonard, A.E., Mukerji, P., 2003. Recent advances in the study of fatty acid desaturases from animals and lower eukaryotes. Prostag. Leukotr. Ess. 68, 97-106.

Reis, D.B., Acosta, N.G., Almansa, E., Navarro, J.C., Tocher, D.R., Monroig, O., Andrade, J.P., Sykes, A.V., Rodríguez, C., 2014a. In vivo metabolism of unsaturated fatty acids in Octopus vulgaris hatchlings determined by incubation with ${ }^{14} \mathrm{C}$-labelled fatty acids added directly to seawater as protein complexes. Aquaculture 431, 28-33.

Reis, D.B., García-Herrero, I., Riera, R., Felipe, B.C., Rodríguez, C., Sykes, A.V., Martín, M.V., Andrade, J.P., Almansa, E., 2014b. An insight on Octopus vulgaris paralarvae lipid requirements under rearing conditions. Aquacul. Nutr. doi: 10.1111/anu.12205.

Sargent, J., McEvoy, L., Estevez, A., Bell, G., Bell, M., Henderson, J., Tocher, D., 1999. Lipid nutrition of marine fish during early development: current status and future directions. Aquaculture 179, 217-229.

Sinanoglou, V.J., Miniadis-Meimaroglou, S., 1998. Fatty acid of neutral and polar lipids of (edible) Mediterranean cephalopods. Food Res. Int. 31, 467-473.

Sinanoglou, V.J., Miniadis-Meimaroglou, S., 2000. Phospholipids in Mediterranean cephalopods. Z. Naturforsch. C 55, 245-255.

Sprecher, H., 2000. Metabolism of highly unsaturated n-3 and n-6 fatty acids. Biochim. Biophys. Acta 1486, 219-231.

Sykes, A.V., Domingues, P.M., Correia, M., Andrade, J.P., 2006a. Cuttlefish culture Stat of the art and future trends. Vie Milieu 56, 129-137.

Sykes, A.V., Domingues, P.M., Andrade, J.P., 2006b. Effects of using grass shrimp (Palaemonetes varians) as the only source of food for the culture of cuttlefish, Sepia officinalis (Linnaeus, 1758). Aquacult. Int. 14, 551-568.

Sykes, A.V., Almansa, E., Lorenzo, A., Andrade, J.P., 2009. Lipid characterization of both wild and cultured eggs of cuttlefish (Sepia officinalis L.) throughout the embryonic development. Aquacult. Nutr. 2009, 38-53. 
Sykes, A.V., Gonçalves, R.A., Andrade, J.P., 2013. Early weaning of cuttlefish (Sepia officinalis, L.) with frozen grass shrimp (Palaemonetes varians) from the first day after hatching. Aquac. Res. 44, 1815-1823.

Sykes, A.V., Domingues, P., Andrade, J.P., 2014. Sepia officinalis in Iglesias, J., Fuentes, L., Villanueva, R., (Eds.) Chephalopod Culture. Springer, Heidelberg, pp. 175-204.

Tocher, D.R., Harvie, D.G., 1988. Fatty acid compositions of the major phosphoglycerides from fish neural tissues: (n-3) and (n-6) polyunsaturated fatty acids in rainbow trout (Salmo gairdneri, L.) and cod (Gadus morhua) brains and retinas. Fish Physiol. Biochem. 5, 299-239.

Tocher, D.R., Leaver, M.J., Hodgson, P.A., 1998. Recent advances in the biochemistry and molecular biology of fatty acyl desaturases. Prog. Lipid Res. 37, 73-117. Wilson, R., Sargent, J.R., 1992. High-resolution separation of polyunsaturated fatty acids by argentation thin-layer chromatography. J. Chromatogr. 623, 403-407. Zar JH., 1999. Biostatistical Analysis. Prentice-Hall, Englewood Cliffs, New Jersey . 
619

620

621

622

623

624

625

626

627

628

629

630

631

632

633

634

635

636

Table 1 - Total lipid content and lipid class composition of Sepia officinalis hatchlings

\begin{tabular}{l}
\hline TL content $\left(\mu \mathrm{g}\right.$ lipid.mg protein $\left.{ }^{-1}\right)$ \\
\hline Lipid Class $(\%)$
\end{tabular}

Sphingomyelin

$0.8 \pm 0.4$

Phosphatidylcholine

$24.0 \pm 1.9$

Phosphatidylserine

$7.0 \pm 0.6$

Phosphatidylinositol

$5.0 \pm 0.2$

Phosphatidylglycerol

$2.4 \pm 0.2$

Phosphotidylethanolamine

$18.9 \pm 1.0$

$\sum$ Polar lipids

$58.1 \pm 1.0$

Cholesterol

$32.2 \pm 2.1$

Free fatty acids

$0.8 \pm 0.2$

Triacylglycerols

$6.3 \pm 1.1$

Sterol esters

$2.6 \pm 0.8$

$\sum$ Neutral lipids

$41.9 \pm 1.0$

Results represent means $\pm \mathrm{SD} ; \mathrm{n}=4$.

Data are presented in percentage of TL content. 
Table 2 - Fatty acid composition ( $\%$ of total

FA) of S. officinalis hatchlings

\begin{tabular}{ll}
\hline $16: 0$ & $20.4 \pm 1.6$ \\
$18: 0$ & $10.9 \pm 0.3$ \\
$\sum$ saturated $^{\mathrm{a}}$ & $42.3 \pm 2.7$
\end{tabular}

\begin{tabular}{|c|c|}
\hline $16: 1^{b}$ & $0.9 \pm 0.0$ \\
\hline $18: 1 n-9$ & $1.5 \pm 0.3$ \\
\hline 18:1n-7 & $3.4 \pm 0.3$ \\
\hline $20: 1 n-9$ & $3.4 \pm 0.1$ \\
\hline$\sum$ monoenes $^{\mathrm{a}}$ & $13.8 \pm($ \\
\hline
\end{tabular}

$18: 2 n-6$

$0.6 \pm 0.1$

$20: 2 \mathrm{n}-6$

$0.3 \pm 0.0$

$20: 4 n-6$

$1.3 \pm 0.4$

$\sum \mathrm{n}-6 \mathrm{FA}^{\mathrm{a}}$

$2.2 \pm 0.3$

$\sum n-6$ LC-PUFA

$1.3 \pm 0.4$

$20: 3 n-3$

$1.3 \pm 0.4$

$20: 5 n-3$

$16.4 \pm 1.0$

$22: 5 n-3$

$1.4 \pm 0.1$

$22: 6 n-3$

$21.5 \pm 1.7$

$\sum \mathrm{n}-3 \mathrm{FA}^{\mathrm{a}}$

$40.9 \pm 2.9$

$\sum \mathrm{n}-3$ LC-PUFA

$40.5 \pm 3.0$

$\sum$ PUFA $^{\mathrm{a}, \mathrm{c}}$

$43.1 \pm 2.6$

$\sum$ LC-PUFA $^{\mathrm{a}, \mathrm{d}}$

$41.8 \pm 2.7$

n-3/n-6

$18.8 \pm 3.4$

DHA/EPA ${ }^{\mathrm{e}}$

$1.3 \pm 0.0$

EPA/ARA ${ }^{\mathrm{e}}$

$13.4 \pm 3.9$

639

Results represent means $\pm S D ; n=4$.

Data are presented in percentage of total FA content.

${ }^{\mathrm{a}}$ Totals include some minor components not shown

${ }^{\mathrm{b}}$ Contain n-9, n-7 and n-5 isomers

${ }^{c}$ PUFA - Polyunsaturated fatty acids

${ }^{\mathrm{d}}$ LC-PUFA - Long-chain polyunsaturated fatty acids

${ }^{\text {e } A R A ~-~ 20: 4 n-6 ; ~ E P A ~-~ 20: 5 n-3 ; ~ D H A ~-~ 22: 6 n-3 ~}$ 
Tamte 3 - Incorporation of radioactivity into total lipid (pmoles.mg $\mathrm{pp}^{-1} \cdot \mathrm{h}^{-1}$ ) and its esterification (\%) into lifink classes of $S$. officinalis hatchlings incubated with $\left[1-{ }^{14} \mathrm{C}\right] \mathrm{FA}$ substrates

\begin{tabular}{|c|c|c|c|c|c|c|}
\hline Substrate & $18: 1 n-9$ & $18: 2 n-6$ & $18: 3 n-3$ & $20: 4 n-6$ & $20: 5 n-3$ & $22: 6 n-3$ \\
\hline Incorporation & $8.2 \pm 1.4^{\mathrm{a} \bullet}$ & $15.4 \pm 3.4^{\mathrm{bc} \boldsymbol{\Lambda}}$ & $18.0 \pm 3.7^{\mathrm{c} \Delta}$ & $18.0 \pm 1.3^{c \Delta}$ & $14.6 \pm 2.9^{\mathrm{bc} o \Delta}$ & $11.4 \pm 1.1^{\mathrm{ab} \circ}$ \\
\hline \multicolumn{7}{|l|}{ Lipid Class (\%) } \\
\hline Phosphatidylcholine & $38.3 \pm 3.0^{\mathrm{c} \bullet}$ & $39.2 \pm 1.7^{\mathrm{bc} \bullet}$ & $44.7 \pm 2.1^{\mathrm{b} \boldsymbol{\Delta}}$ & $26.9 \pm 1.1^{\mathrm{do}}$ & $26.7 \pm 0.5^{\mathrm{do}}$ & $70.6 \pm 4.8^{\mathrm{a} \Delta}$ \\
\hline Phosphatidylserine & $25.2 \pm 1.4^{\mathrm{a} \boldsymbol{\Delta}}$ & $22.2 \pm 1.4^{\mathrm{b} \bullet}$ & $21.5 \pm 0.5^{\mathrm{bc} \bullet}$ & $19.3 \pm 1.4^{\operatorname{cd} \Delta}$ & $17.2 \pm 1.4^{\mathrm{d} \Delta}$ & $2.9 \pm 0.3^{\mathrm{eo}}$ \\
\hline Phosphatidylinositol & $4.1 \pm 0.9^{c}$ & $3.2 \pm 0.5^{\mathrm{c}}$ & $3.4 \pm 0.1^{\mathrm{c}}$ & $11.1 \pm 1.4^{\mathrm{a} \Delta}$ & $11.5 \pm 0.9^{\mathrm{a} \Delta}$ & $6.1 \pm 1.4^{\text {bo }}$ \\
\hline Phosphatidylglycerol & $0.0 \pm 0.0^{\mathrm{c}}$ & $3.90 .3^{\mathrm{a} \Lambda}$ & $3.30 .2^{b \bullet}$ & $0.0 \pm 0.0^{\mathrm{c}}$ & $0.0 \pm 0.0^{\mathrm{c}}$ & $0.0 \pm 0.0^{\mathrm{c}}$ \\
\hline Phosphatidylethanolamine & $12.9 \pm 0.8^{\mathrm{b} \bullet}$ & $15.0 \pm 0.8^{\mathrm{b} \boldsymbol{\Delta}}$ & $14.2 \pm 1.1^{\mathrm{b} \boldsymbol{\bullet} \bullet}$ & $36.6 \pm 2.2^{\mathrm{a} \Delta}$ & $37.5 \pm 1.9^{\mathrm{a} \Delta}$ & $12.6 \pm 1.0^{\mathrm{b} \circ}$ \\
\hline$\sum$ Polar lipids & $80.6 \pm 3.4^{\mathrm{d} \bullet}$ & $83.5 \pm 1.9^{\operatorname{cd} \Lambda \bullet}$ & $87.1 \pm 3.1^{\text {bc } \Lambda}$ & $93.9 \pm 2.0^{\mathrm{a}}$ & $92.9 \pm 1.5^{\mathrm{a}}$ & $92.2 \pm 2.5^{\mathrm{ab}}$ \\
\hline Partial acylglyce & $5.4 \pm 2.2^{\mathrm{a}}$ & $5.1 \pm 0.5^{\mathrm{a}}$ & $3.3 \pm 1.2^{\mathrm{ab}}$ & $2.1 \pm 0.7^{\mathrm{b}}$ & $2.1 \pm 0.7^{b}$ & $2.9 \pm 1.1^{\mathrm{ab}}$ \\
\hline Free fatty acids & $2.3 \pm 0.9$ & $2.1 \pm 1.0$ & $1.9 \pm 0.7$ & $0.7 \pm 0.3$ & $0.9 \pm 0.3$ & $1.5 \pm 1.1$ \\
\hline Triacylglycerols & $11.7 \pm 1.3^{\mathrm{a} \boldsymbol{\Delta}}$ & $9.3 \pm 1.5^{\mathrm{ab} \boldsymbol{\Delta} \bullet}$ & $7.7 \pm 1.2^{\mathrm{b} \bullet}$ & $3.3 \pm 1.1^{\mathrm{c}}$ & $4.1 \pm 1.3^{c}$ & $3.4 \pm 0.6^{c}$ \\
\hline$\sum$ Neutral lipids & $19.4 \pm 3.4^{\mathrm{a} \boldsymbol{\Lambda}}$ & $16.5 \pm 1.9^{\mathrm{ab} \Lambda \bullet}$ & $12.9 \pm 3.1^{\mathrm{bc} \bullet}$ & $6.1 \pm 2.0^{\mathrm{d}}$ & $7.1 \pm 1.5^{\mathrm{d}}$ & $7.8 \pm 2.5^{\mathrm{cd}}$ \\
\hline
\end{tabular}

BASAlts represent means $\pm \mathrm{SD} ; \mathrm{n}=4$

D5G of incorporation are presented in pmoles of ${ }^{14} \mathrm{C}$ fatty acid incorporated /mg protein per hour

D5th of esterification are given in percentage

B5Ferent letters in superscript within the same row represent significant differences within all fatty acids $(p<0.05)$

B5ß3erent full symbols in superscript $\left({ }^{\mathbf{\Delta} \bullet}\right)$ within the same row represent significant differences within C18 FA $(p<0.05)$

D5fferent hollow symbols in superscript $\left({ }^{\Delta \circ}\right)$ within the same row represent significant differences within LC-PUFA (655).05).

656 
660
Table 4 - Recovery of radioactivity from $\left[1-{ }^{14} \mathrm{C}\right] \mathrm{FA}$ substrates in FA metabolites in S. officinalis hatchlings

\begin{tabular}{|c|c|}
\hline Fatty acids & $\%$ recovery \\
\hline \multicolumn{2}{|l|}{$\left[1-{ }^{14} \mathrm{C}\right] 18: 1 \mathrm{n}-9$} \\
\hline $18: 1 n-9$ & $43.2 \pm 7.3$ \\
\hline $20: 1 n-9$ & $40.0 \pm 5.6$ \\
\hline $22: 1 n-9$ & $12.1 \pm 1.9$ \\
\hline $18: 2 n-9$ & $4.7 \pm 0.7$ \\
\hline \multicolumn{2}{|l|}{$\left[1-{ }^{14} \mathrm{C}\right] 18: 2 \mathrm{n}-6$} \\
\hline $18: 2 n-6$ & $48.3 \pm 1.7$ \\
\hline $20: 2 n-6$ & $43.4 \pm 1.8$ \\
\hline $22: 2 n-6$ & $5.9 \pm 0.6$ \\
\hline $18: 3 n-6$ & $1.3 \pm 0.2$ \\
\hline UK & $1.1 \pm 0.1$ \\
\hline \multicolumn{2}{|l|}{$\left[1-{ }^{14} \mathrm{C}\right] 18: 3 \mathrm{n}-3$} \\
\hline $18: 3 n-3$ & $58.5 \pm 6.3$ \\
\hline $20: 3 n-3$ & $36.2 \pm 5.8$ \\
\hline $22: 3 n-3$ & $3.6 \pm 0.7$ \\
\hline $18: 4 n-3$ & $1.7 \pm 0.2$ \\
\hline \multicolumn{2}{|l|}{$\left[1-{ }^{14} \mathrm{C}\right] 20: 4 n-6$} \\
\hline $20: 4 n-6$ & $96.9 \pm 1.2$ \\
\hline $22: 4 n-6$ & $2.1 \pm 1.2$ \\
\hline UK & $1.0 \pm 0.0$ \\
\hline \multicolumn{2}{|l|}{$\left[1-{ }^{14} \mathrm{C}\right] 20: 5 \mathrm{n}-3$} \\
\hline $20: 5 n-3$ & $98.3 \pm 0.3$ \\
\hline $22: 5 n-3$ & $1.7 \pm 0.3$ \\
\hline \multicolumn{2}{|l|}{$\left[1-{ }^{14} \mathrm{C}\right] 22: 6 \mathrm{n}-3$} \\
\hline $22: 6 n-3$ & $94.5 \pm 1.3$ \\
\hline $24: 6 n-3$ & $5.5 \pm 1.3$ \\
\hline
\end{tabular}

